\title{
Kinetics and Kinematics of \\ Sprinting in Mid and Post \\ Peak Height Velocity Female
}

\section{Athletes}

Kaushik Talukdar, ${ }^{1,2,3}$, Dr Craig Harrison², Professor Mike McGuigan ${ }^{3,4}$, Dr Robert Borotkanics ${ }^{3}$

'St Cuthbert's College, Auckland, New Zealand; '2Sports Performance Research Institute of New Zealand, Auckland, New Zealand; ${ }^{3}$ Faculty of Health and Environmental Sciences, Auckland University of Technology; ${ }^{4}$ School of Medical and Health Sciences Edith Cowan University, Australia; ${ }^{5}$ Athlete Institute, Auckland, New Zealand

\section{ABSTRACT}

Sprinting speed is a crucial physical capacity and can change throughout an athlete's growth. Previous research has shown that both kinetic and kinematic variables change across maturation in young males. However, due to the changes in growth and hormonal levels, the kinetic and kinematic factors associated with sprinting may vary in young females compared to their male counterparts. Therefore, identifying the natural development of sprinting kinetics (force, maximal power) and kinematics (step length, step frequency, contact time and flight time) in young females can provide valuable insights into training for this cohort. Thirty-two young female athletes, 11 mid-peak height velocity (PHV) age $(12.8 \pm 0.6)$ and 21 post PHV (13.5 \pm 0.93$)$ performed two 15 and $30 \mathrm{~m}$ sprints each. Theoretical velocity, maximal velocity, step length, force and power max were significantly higher in post PHV girls $(p<0.05)$. Univariate regression analysis reported that the best predictors of velocity ( 15 and $30 \mathrm{~m}$ ) were contact time, power max, stride frequency, step length and leg length with contact time being the strongest predictor. The findings of this research provide insight into the natural development of sprinting in young females and will help practitioners specifically develop training programs that can effectively improve sprinting kinetics and kinematics in this cohort.

Keywords: sprinting, youth, female, kinetics, kinematics

\section{INTRODUCTION}

Running velocity in is determined by several factors such as anthropometry (measurements and proportions of the body), kinetics (horizontal and vertical forces) and kinematics (step length, frequency, contact and flight time) (Rumpf et al., 2015; Meyers et al., 2015; Meyers et al., 2017b). Most studies that have investigated kinetic (horizontal and vertical forces) and kinematic (step length, step frequency, contact time, and flight time) variables of sprinting speed have been conducted in adult populations (Simperingham et al., 2017; Brughelli, Cronin, \& Chaouachi, 2011; Nilsson \& Thorstensson, 1989; Nummela, Keranen, \& Mikkelsson, 2007) with limited studies on youth (Schepens, Willems, Cavagna, 1998; Rumpf, et al., 2015; Meyers et al., 2015; Meyers et al., 2017a; Nagahara et al., 2019). Due to growth, maturity and changes in anthropometry, the interaction between kinetic and kinematic variables can play a significant role in sprinting speed in youth across maturation (Rumpf et al., 2015).

It has been reported that sprinting speed in boys and girls tends to develop in a non-linear fashion throughout childhood and adolescence (Viru et al., 1999) with accelerated development of sprint performance during both preadolescent and adolescent periods (Meyers et al., 2015; Viru et al.,1999). Furthermore, according to the Youth Physical Development (YPD) Model speed training 
can be broken down into three different stages (prior to late adolescence stage): early childhood (age 0-7 years), prepubertal (age 7-12 years), circumpubertal (age 11-15 years males, age 12-15 years females) and late adolescence (age 16+ years males, age 15+ years females) (Lloyd \& Oliver, 2012; Oliver, Lloyd \& Rumpf, 2013). Even if the chronological reference regarding speed development windows can provide flexibility in training, it is important to consider PHV as it coincides with the development of speed during growth spurts (Philippaerts et al., 2006). Therefore, natural development of speed may be maturity dependent and requires further investigation (Meyers et al., 2015; Rumpf et al., 2015).

Limited studies have investigated the role of maturity in the kinetic and kinematic variables of sprinting speed among youth. Rumpf et al. (2015) found significant differences in kinetic (horizontal and vertical force) and kinematic variables (step length and frequency) in young male athletes across maturation. More specifically, sprinting kinetics and kinematics in pre PHV athletes differed 8-78\% compared to mid and post PHV athletes with the greatest average change found between pre and mid PHV athletes (37.8\%) compared to (11.6\%) between mid and post PHV athletes. Similarly, Meyers et al. (2015) reported significant differences in stride length between pre and mid PHV boys but not between mid and post PHV boys over 30 meters $(p<0.05)$. With regards to contact time, a significant difference was reported between early pre PHV boys but not between mid and post PHV boys $(p<0.05)$ (Meyers et al. 2015). Meyers et al. (2016) also reported significantly greater increases in speed $(10.4 \%$ vs $5.6 \%)$, relative vertical stiffness $(12.1 \% \vee 5.6 \%)$ in pre to post boys compared to pre PHV boys $(p<0.05)$. In addition, stride frequency and contact time seem to be significantly different between early pre PHV and pre PHV boys but not mid and post PHV boys $(\mathrm{p}<0.05)$ (Meyers et al., 2015; Meyers et al., 2016). This could be because maximum sprint speed appears to develop around mid and post PHV phase when stride frequency and contact time begin to stabilize (Meyers et al., 2015). In addition, boys who are early pre PHV may lack motor coordination and strength to effectively orientate, stabilise and apply force through their limbs compared to mid and post PHV boys (Meyers et al., 2015).

Furthermore, it is hypothesised that due to rise of testosterone and growth hormones during puberty (Ramos et al., 1998; Forbes et al., 2009), improvements in strength and power output may affect force production (Armstrong et al., 2000; Forbes et al., 2009). Hence, kinetics and kinematics of running may differ in participants of varying maturity status. However, the secondary sex characteristics caused by an increased secretion of growth hormones during puberty, can increase fat mass in girls compared to boys (Malina et al., 2004). This can inhibit force production and negatively affect sprinting speed (Nagahara et al., 2019). In addition, studies have reported a negative relationship between body fat mass and kinematic variables of sprinting speed such as step length, and step frequency in post and pre PHV boys respectively (Meyers et al., 2017a). Therefore, it is important to investigate sprinting kinetics and kinematics across maturation in girls.

It appears that limited studies have investigated kinetics and kinematics of sprinting speed in young females across age groups (Nagahara et al., 2019; Vanderka \& Kampmiller, 2012). Furthermore, previous studies did not include maturity offset and divided girls based on their chronological age (Nagahara et al., 2019; Vanderka \& Kampmiller, 2012). Due to the disparity in variables associated with sprinting speed across age groups, it is important to consider maturity offset to determine the changes in kinetic and kinematic variables of sprinting speed in young females. Nagahara et al. (2019) reported significant changes in sprinting speed between younger ( $<12.7$ years) and older girls (>12.7 years). The older girls in this study were significantly quicker than the younger girls for $25 \mathrm{~m}$ and $50 \mathrm{~m}$ sprints $(p<0.05)$. However, the step length over maximal sprint and propulsive forces during acceleration were significantly greater $0.08 \mathrm{~m} / \mathrm{y}$ and $0.024 \mathrm{Ns} /$ in the younger girls compared to 0.01 $\mathrm{m} / \mathrm{y}$ and $-0.010 \mathrm{Ns} / \mathrm{y}$ in the older girls. Furthermore, Vanderka and Kampmiller (2012) reported a stagnation in sprinting speed after 14 to 15 years in girls compared to boys. The differences in sprinting kinetics and kinematics in the previous studies may be due to the greater growth rates in the younger girls and greater fat mass with maturation in older girls (Nagahara et al., 2019).

In addition, a recent study that investigated ground reaction forces (GRFs) in sprinting in both boys and girls (untrained) reported that the increase in maximal velocities in pre PHV girls (2.5-1.5 years before PHV) compared to pre PHV boys (1.5-0.5 years from PHV) were not attributed to increase in GRFs but rather due to longer ground contact time Effect size (ES): \pm 90\% Confidence Interval (Cl) 
$=1.00 \pm 0.78$. (Coyler et al., 2020). However, the study included non-athletic pre PHV girls that may not provide relevant insights into sprinting kinetics and kinematics across maturation, particularly in mid and post PHV female athletes. Therefore, the purpose of this study was to determine the kinetics and kinematics of sprinting speed in mid and post PHV female athletes and investigate their relationship with maximal velocity. It is hypothesised that there will be significant differences in kinetics and kinematics between mid and post PHV female athletes and both kinetic and kinematic variables will influence maximal velocity in this cohort.

\section{METHODS}

\section{Participants}

Thirty- two female athletes (11 mid PHV and 21 post PHV) from sports teams (hockey, football, and netball) with a minimum training age of 1 year in their respective sports at a private girl's college in New Zealand volunteered for this study. All participants were healthy and without any reported injuries in the last 3 months. The study was approved by the Auckland University of Technology Ethics Committee. All participants and their legal guardians were informed of the risks and benefits of participation and both legal guardians and participants provided written consent and assent to participate in this study. The participants' characteristics are provided in table 1.

Anthropometric measurements and date of birth were taken before familiarisation, including height $(\mathrm{m})$, sitting height $(\mathrm{m})$, leg length $(\mathrm{cm})$ and weight $(\mathrm{kg})$. Maturity status of the participants was calculated using the Mirwald and colleagues' equation (2002). This method is considered non-invasive and predicts years from PHV as a measure of maturity offset using anthropometric variables. Participants are generally classified into three groups as follows pre PHV velocity ( -3 years to -1 years from PHV), mid PHV $(-1$ to +1 years from PHV), and post PHV $(+1$ to +3 years from PHV) (Rumpf et al., 2012). The equation for maturity offset for girls was:

Maturity Offset for girls $=-9.376+0.0001882 \cdot \mathrm{Leg}$ Length and Sitting Height interaction $+0.0022 \cdot$ Age and Leg Length interaction + 0.005841.Age and Sitting Height interaction - 0.002658 Age and Weight interaction $+0.07693 \cdot$ Weight by Height ratio 100

\section{Procedures}

Participants were required to attend three sessions. First, a familiarisation session was conducted 2 days before any data were collected, consisting of various intensity sprints. Then, participants attended a second session during which their sprinting speed over a distance of $30 \mathrm{~m}$ was assessed using a radar gun (Version 5.0.2.1. Applied Concepts, Inc, Texas, USA) to determine kinetic variables including force (Fo), maximal power (Pmax), and velocity (Vo). Participants sprinted from a static split stance position with their leading foot immediately behind the start line. The radar gun with a sampling rate of $47 \mathrm{~Hz}$, placed $5 \mathrm{~m}$ directly behind the start line, was used to measure sprinting speed. The operating range of the gun was set at $0 \mathrm{~m} / \mathrm{s}$ (low-from zero acceleration starting position) to $14 \mathrm{~m} / \mathrm{s}$ (hightypical top end speed that is not surpassed). The gun was set on a tripod set at $0.9 \mathrm{~m}$ above ground to approximately align with the centre of mass of the participants (Morin et al., 2006). No false start was allowed and participants were instructed to sprint maximally to a fixed marker $5 \mathrm{~m}$ past the $30 \mathrm{~m}$ mark (Simperingham et al., 2017). Participants performed two maximal sprints separated by 5 min of passive rest and the best of the two based on all dependent variables (Fo, Pmax, and Vo) were taken for analysis.

Table 1. Characteristics (Mean \pm SD) of participants across maturity groups

\begin{tabular}{lccccc}
\multicolumn{1}{c}{ Groups } & Age (years) & $\begin{array}{c}\text { Maturity Offset } \\
\text { (years from } \\
\text { PHV) }\end{array}$ & Height $(\mathbf{m})$ & Leg length(cm) & $\begin{array}{c}\text { Body mass } \\
\mathbf{( k g )}\end{array}$ \\
\hline Mid- PHV & $12.70 \pm 0.56$ & $0.58 \pm 0.35$ & $1.56 \pm 0.07$ & $85.14 \pm 5.02$ & $42.05 \pm 6.75$ \\
Post- PHV & $13.53 \pm 0.91$ & $1.82 \pm 0.50$ & $1.66 \pm 0.04$ & $91.40 \pm 3.36$ & $54.79 \pm 7.64$ \\
\hline
\end{tabular}

${ }^{*}$ Significant difference $(p<0.05)$ in leg length, height and body mass 
Horizontal velocity was measured continuously using the radar device connected to a laptop running Stalker ATS System software (Version 5.0.2.1, Applied Concepts Inc, Texas, USA) (Simperingham et al., 2017). The raw data files were automatically processed using the digital filter "dig light". This function is available within the software and precisely removes noise frequencies while preserving data frequency being measured. The dig light filter applies minimal filtering and suitable for "clean" radar data and applies a fourth order (one round trip), Butterworth low-pass zero lag filter with a cut off frequency of $8 \mathrm{~Hz}$. To improve consistency all trials were nominated to be acceleration runs hence forcing the start of the velocity-time curve through the zero point (Simperingham et al., 2017). The processed data were then imported into a custommade Lab View (Version 13.0, National Instruments, Corporation, Texas, USA) to calculate all outcome variables (Fo, Vo, Pmax, Vmax and split times between 0 and $30 \mathrm{~m}$ ) (Buchheit et al., 2014; Cross et al., 2015; Morin \& Seve, 2011). The velocity-time curve $[\mathrm{v}(\mathrm{t})]$ for each sprint was calculated using the exponential function $\mathrm{v}(\mathrm{t})=V \max \times(1-\mathrm{e}-\mathrm{t} \tau)$ (Haddad, Simpson, \& Buchheit, 2015), horizontal acceleration was calculated from Newton's second law of motion $\mathrm{Fh}(\mathrm{t})=[\mathrm{m} \times \mathrm{a}(\mathrm{t})]+$ Fair $(\mathrm{t})$ (Arsac \& Locatelli, 2002) and Pmax was calculated through the equation Pmax $=\left(0.5 \times F_{O}\right) \times(0.5 \times$ Vo $)($ Bezodis et al., 2012). Data recorded for both the trials were used in the assessment of intra-day and the best trials on each for the inter-day reliability. A moderate to strong ICC $=0.74-0.98$ with a $\mathrm{CV}$ ranging from $1.70-12.70 \%$ across all kinetic variables (Fo, $V_{0}$, Vmax, Pmax, 10, 20, and $30 \mathrm{~m}$ split times) were reported for both intraday and inter-day reliability in this population (Talukdar et al., 2021).

The third session involved participants sprinting over a distance of $15 \mathrm{~m}$ to assess kinematic variables including step length, step frequency, flight time, contact time and velocity max using the Optojump Next System (Microgate, Bolzano, Italy) positioned at floor level. No false start was allowed and participants were instructed to sprint maximally to a fixed marker $5 \mathrm{~m}$ past the $15 \mathrm{~m}$ mark (Meyers et al., 2015). Data for all the sprinting kinematic characteristics were collected using a Windows laptop via Optojump software (Microgate, Italy) and later exported to Microsoft Excel for processing (Meyers et al., 2015). The reliability between trials for all kinematic variables in this study were high with ICC ranging from 0.81-0.94 and CV ranging from 1.7-5.7\%. Previous researchers have also reported strong ICC $=0.87-0.98$ and $C V=0.6-5.5 \%$ in measuring stride characteristics in the adult population using the Optojump (Oliver \& Stembridge, 2011). Kinematic variables (step length, step frequency, contact time, and maximal velocity for $15 \mathrm{~m}$ ) were derived from the Optical measurement system and kinetic variables (Fo, Pmax and Vo over $30 \mathrm{~m}$ ) were derived using the radar gun were defined as follows (Meyers et al., 2015; Simperingham et al., 2017):

Step length $(\mathrm{cm})$ : distance covered during the flight phase over one step

Normalised step length $(\mathrm{cm})$ : step length divided by leg length

Step frequency $(\mathrm{Hz})$ : 1/contact time + aerial time of a step length

Contact time (s): the amount of time (s) the participant spends during the stance phase of the sprint where the foot is in contact with the floor

Flight time (s): the amount of time (s) between foot contacts, where the participant is not in contact with the floor

Pmax (W): Peak horizontal forces $\times$ velocity

$\operatorname{Vmax}(\mathrm{m} / \mathrm{s})$ : Maximum speed attained for the distance $15 \mathrm{~m}$ by the participant

Vo $(\mathrm{m} / \mathrm{s})$ : theoretical velocity max attained by the participant over the distance of $30 \mathrm{~m}$

Fo (N): Horizontal forces associated with sprinting over the distance of $30 \mathrm{~m}$

All the sessions began with a standardised warmup, including $20 \mathrm{~m}$ multi-directional runs (forward, backward, shuffle and crossover), dynamic stretching and a series of sub-maximal sprints $(50 \%$, $75 \%, 90 \%$ effort). Five minutes and 2.5 minutes of passive rest were given between 30 and $15 \mathrm{~m}$ sprints, respectively.

\section{Statistical Analysis}

Means and the standard deviation (SD) were used for all dependent variables of interest as measures of centrality and spread of data using SPSS V.25 (SPSS Inc, Chicago, IL, USA). Tests of model assumptions (conditional SD, mean and distribution) were carried out. Univariate regressions were conducted to a) to detect changes across variables between both groups and b) identify which kinematic and kinetic factors were important in predicting maximum velocity when maturity offset was controlled. Regression analysis was also used to determine whether any outliers were present and normal distribution of data. An alpha level of 0.05 was used for all statistical tests. 


\section{Results}

There was a significant difference between the groups with regards to height, bodyweight and leg length (Table 1). Mean values of the variables of interest and their percentage change are reported in table 2.

In terms of kinematic variables, maximum velocity for the $15 \mathrm{~m}$ (5.19\%) and step length (7.95\%) were significantly higher $(\mathrm{p}<0.05)$ in post PHV girls (Table 2). However, when step length was divided by leg length there was no difference between the groups. Furthermore, there were no significant differences between the groups with regards to contact time and flight time. However, mid PHV girls had a marginally (non-significant) higher step frequency compared to post PHV girls. With regards to kinetic variables, it was found that Vo over $30 \mathrm{~m}$ (10\%), Fo (50.39\%) and Pmax (57.90\%) were significantly higher in post PHV girls compared to mid PHV girls, as shown in table 2.

When maturity offset was controlled, regression analysis reported the predictors of velocity over 15 $\mathrm{m}$ were contact time, Pmax, step frequency, and step length. Whereas the predictors of velocity over $30 \mathrm{~m}$ were contact time, leg length and Pmax. In addition, out of all the variables, contact time and
Pmax predicted maximal velocity for both 15 and $30 \mathrm{~m}$ sprints. However, contact time was the best predictor of sprint velocity for both 15 and $30 \mathrm{~m}$ sprints, followed by step frequency for $15 \mathrm{~m}$ and leg length for $30 \mathrm{~m}$ (Table 3). Moreover, Pmax and step length were not the strongest predictors with regards to magnitude but the standard error was comparatively lower $(\leq 0.005)$ across variables (Table 3). Interpretation of the regression analysis is provided in Table 3 for each variable. The regression model of contact time its association with $30 \mathrm{~m}$ maximal velocity is shown in Figure 1.

Table 2. Means, standard deviation and differences for all variables between maturity groups

\begin{tabular}{|c|c|c|c|c|c|}
\hline Variables & MID PHV & POST PHV & $\operatorname{Min}(95 \% \mathrm{Cl})$ & $\operatorname{Max}(95 \% \mathrm{Cl})$ & $\%$ Difference \\
\hline Contact time (s) & $0.17 \pm 0.02$ & $0.17 \pm 0.02$ & -0.01 & 0.02 & 2.83 \\
\hline Flight time (s) & $0.10 \pm 0.01$ & $0.10 \pm 0.01$ & -0.01 & 0.01 & 2.59 \\
\hline $\begin{array}{l}\text { Step length } \\
\text { (cm) }\end{array}$ & $131.22 \pm 9.43$ & $141.66 \pm 10.21$ & 2.87 & 18.01 & $7.95^{\star}$ \\
\hline $\begin{array}{l}\text { Step frequency } \\
(\mathrm{Hz})\end{array}$ & $3.78 \pm 0.26$ & $3.68 \pm 0.27$ & -0.31 & 0.10 & -2.65 \\
\hline $\begin{array}{l}\text { Normalized } \\
\text { step length } \\
\text { (Step length/leg } \\
\text { length) }(\mathrm{m} / \mathrm{m})\end{array}$ & $1.54 \pm 0.10$ & $1.55 \pm 0.12$ & -0.08 & 0.09 & 0.65 \\
\hline $\begin{array}{l}15 \mathrm{~m} V \max \\
(\mathrm{m} / \mathrm{s})\end{array}$ & $5.20 \pm 0.33$ & $5.47 \pm 0.25$ & 0.06 & 0.49 & $5.19^{*}$ \\
\hline $30 \mathrm{~m}$ Vo $(\mathrm{m} / \mathrm{s})$ & $6.73 \pm 0.60$ & $7.40 \pm 0.56$ & 0.22 & 1.11 & $9.96^{*}$ \\
\hline Fo $(\mathrm{N})$ & $201.24 \pm 40.99$ & $302.64 \pm 60.53$ & 59.36 & 143.44 & $50.39^{*}$ \\
\hline$P \max (\mathrm{W})$ & $336.91 \pm 63.3$ & $\begin{array}{c}531.97 \pm \\
128.72\end{array}$ & 110.48 & 279.64 & $57.90^{\star}$ \\
\hline
\end{tabular}

${ }^{*}$ Significantly different between groups $\mathrm{p}<0.05$, Vo- theoretical velocity, Fo- Force, Pmax- Power max, Cl- Confidence intervals 
Table 3. Predictor variables for velocity ( 15 and $30 \mathrm{~m}$ ) when maturity was controlled

\begin{tabular}{lcccccc} 
Variables & B & SE & T & P & Min (95\% Cl) & $\begin{array}{c}\text { Max (95\% } \\
\text { Cl) }\end{array}$ \\
\hline CT $(15 \mathrm{~m})$ & -7.001 & 2.633 & -2.662 & 0.0125 & -12.395 & -1.624 \\
CT $(30 \mathrm{~m})$ & -11.416 & 5.784 & -1.974 & 0.058 & -23.245 & 0.413 \\
Pmax (15 m) & 0.001 & 0.000 & 3.085 & 0.004 & 0.000 & 0.002 \\
Pmax (30m) & 0.003 & 0.001 & 2.817 & 0.009 & 0.001 & 0.005 \\
SF (15 m) & 0.379 & 0.183 & 2.074 & 0.047 & 0.005 & 0.753 \\
LL (30 m) & 0.050 & 0.028 & 1.810 & 0.081 & -0.007 & 0.107 \\
SL (15 m) & 0.010 & 0.005 & 2.077 & 0.047 & 0.000 & 0.020 \\
\hline
\end{tabular}

${ }^{*}$ CT- Contact time, Pmax- power max, SF- step frequency, LL- leg length,

SL- step length, B-beta, SE- standard error, Cl- confidence intervals

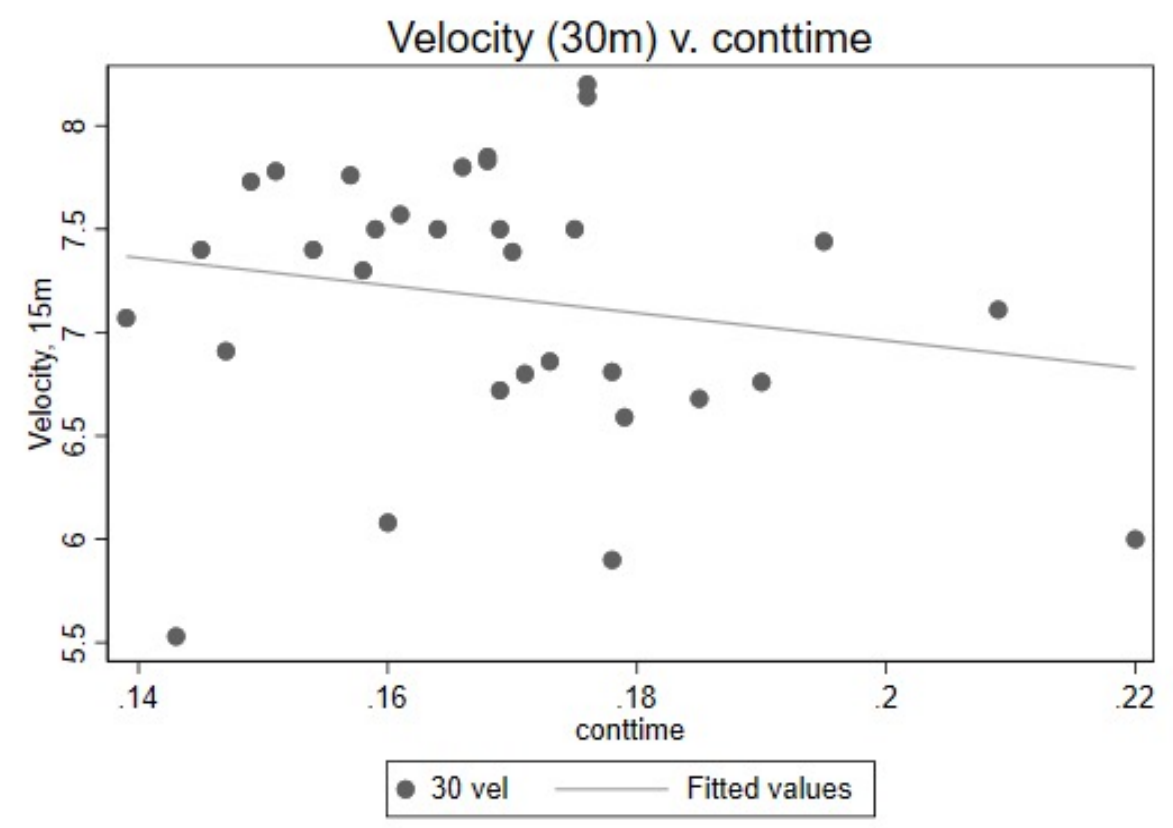

Figure 1. Regression model: Maximal velocity and Contact time

\section{DISCUSSION AND IMPLICATION}

The primary purpose of this study was to determine if the kinematics and kinetics associated with maximum sprinting velocity differ in female youth across maturation. Furthermore, it was also crucial to investigate if the kinematic and kinetic variables such as step length, step frequency, flight time, contact time, horizontal force and Pmax can predict maximal sprinting velocity across maturation in this cohort. The differences in kinetic and kinematic variables associated with maximal sprinting velocity can provide practical insights to coaches working with young female athletes. This study supports previous research that reported an increase in maximal velocity $(15 \mathrm{~m}$ and $30 \mathrm{~m})$ with maturation in youth populations $(\mathrm{p}<0.05)$ (Schepens, Willems, \& Cavagna, 1998; Rumpf et al., 2015; Meyers et al.,
2015; Meyers et al., 2016; Nagahara et al., 2019). This study also found that not all kinematic and kinetic variables measured were strong predictors of maximal velocity when maturity was controlled in this population.

With regard to kinematic variables, step length increased with maturity $(p<0.05)$. However, when step length was divided by leg length (normalised step length) there was no difference between the groups. Rumpf et al. (2015) also found no significant difference in normalised step length between mid and post PHV boys. Similar to previous research (Rumpf et al., 2015; Meyers et al., 2015) this study did not find significant differences in contact time, flight time and step frequency between mid and post PHV groups. A possible explanation for this finding could be that contact time, flight time and step 
frequency tend to stabilise during mid and post PHV phases as greater changes tend to happen during the transition from pre to mid PHV phase (Meyers et al., 2015; Meyers et al., 2016; Rumpf et al., 2015). Therefore, maturity related improvements in strength and power output may enhance improvement in technical efficiency and force application, resulting in improved speed in post PHV females (Forbes et al., 2009; Ramos et al.,1998; Meyers et al., 2015).

The relative horizontal force $(F o$ ) output over $30 \mathrm{~m}$ sprint was significantly different between mid and post PHV groups, with an average change of $>50 \%$ (Table 2). This finding contrasts with those of Rumpf et al. (2015) study, which did not show significant differences between mid and post PHV boys in horizontal force but showed significant differences in vertical force $(p<0.05)$. Therefore, suggesting that changes in horizontal forces between mid and post PHV groups might be greater in over ground sprinting as opposed to non-motorised treadmill.

Similar to Rumpf et al. (2015), power output for the $30 \mathrm{~m}$ sprint was significantly different between mid PHV (337 W) and post PHV (532 W) participants in the present study. Since power was calculated as horizontal force multiplied by velocity, and a significant difference in horizontal force existed between groups, it would appear that horizontal force can significantly influence Pmax (Rumpf et al., 2015). However, the values in this study were modest compared to the Rumpf et al. (2015) study. This may be due to the differences in anthropometry, girls in this study had a lower body mass and height compared to the boys in Rumpf et al. (2015) study. Natural growth along with higher androgen levels with a greater anabolic effect in mid and post PHV boys could have played a role in greater maximal power compared to the girls in this study (Malina et al., 2004; Viru et al., 1999).

In agreement with previous research (Rumpf et al., 2015; Meyers et al., 2015), this study found step length to be a predictor of maximal velocity over 15 $\mathrm{m}$. When maturity offset was controlled, for every $\mathrm{cm}$ increase in step length there was an increase of 0.010 $\mathrm{m} / \mathrm{s}$ in maximal velocity. This could be attributed to leg length since there was an increase of $0.050 \mathrm{~m} / \mathrm{s}$ in velocity with every $\mathrm{cm}$ increase in leg length over the distance of $30 \mathrm{~m}$. This finding suggests that step length and leg length can influence maximal velocity over both 15 and $30 \mathrm{~m}$ respectively. Therefore, measuring step length and leg length can be crucial in investigating sprinting kinematics and maximal velocity in mid and post PHV girls.

In contrast to previous research (Rumpf et al., 2015; Meyers et al., 2015), this study showed step frequency as one of the predictors of maximal velocity over $15 \mathrm{~m}$ but not for $30 \mathrm{~m}$. The maximal velocity in this study increased by $0.379 \mathrm{~m} / \mathrm{s}$ with every $\mathrm{Hz}$ increase in step frequency. Previous study found step frequency to be a better predictor of maximal velocity in pre PHV boys compared to post PHV boys over $30 \mathrm{~m}$ (accounting for $58 \%$ variances in speed) (Meyers et al., 2017a). This could be due to the fact that this study investigated the relationship between step frequency and maximal velocity over the first $15 \mathrm{~m}$ unlike previous studies in boys (Rumpf et al., 2015; Meyers et al., 2015; Meyers et al, 2017a). It has been reported in male sprinters previously that step frequency is crucial in the initial phase of acceleration (Nagahara et al., 2014). Therefore, increasing step frequency in the initial phase of acceleration may be useful in improving maximal velocity in mid and post PHV girls.

Out of all the kinematic variables, contact time was found to be the best predictor of maximal velocity based on the model. Maximal velocity over 15 and $30 \mathrm{~m}$ decreased by $-7.001 \mathrm{~m} / \mathrm{s}$ and $-11.416 \mathrm{~m} / \mathrm{s}$, respectively, with every second increase in contact time. If the regression model is applied contextually, then a $10 \%$ increase in mean contact time across mid and post PHV groups (0.17s) reported in this study will decrease maximal velocity by $0.12 \mathrm{~m} / \mathrm{s}$ (2.23\%) for $15 \mathrm{~m}$ and $0.19 \mathrm{~m} / \mathrm{s}$ (2.69 \%) for $30 \mathrm{~m}$ respectively based on the mean value of maximal velocity across both the groups. Previous research has also reported contact time to be a strong predictor of sprinting speed $\left(\mathrm{R}^{2}=0.70\right)$ in mid PHV boys (Rumpf et al., 2015). Therefore, reducing contact time in young females and males can be useful in improving maximal sprinting velocity.

With regards to kinetic variables, Pmax predicted velocity in this study across $15 \mathrm{~m}$ and $30 \mathrm{~m}$ distances when maturity offset was controlled. The velocity for 15 and $30 \mathrm{~m}$ distances increased by $0.001 \mathrm{~m} / \mathrm{s}(15$ $\mathrm{m})$ and $0.003 \mathrm{~m} / \mathrm{s}(30 \mathrm{~m})$ respectively with every watt increase in power. Similar to the contact time, If the regression model is applied contextually then a $10 \%$ increase in power $(43.44 \mathrm{~W})$ based on the mean score $(434.44 \mathrm{~W})$ for mid and post PHV groups will increase maximal velocity by $0.04 \mathrm{~m} / \mathrm{s}(0.82 \%)$ for 15 $\mathrm{m}$ and $0.13 \mathrm{~m} / \mathrm{s}(1.84 \%)$ for $30 \mathrm{~m}$ respectively. This finding was consistent with previous research that reported power to be a strong predictor of maximal 
velocity in post PHV boys with $R^{2}$ value of 0.35 (Rumpf et al., 2015). However, Rumpf et al. (2015) reported a greater $\mathrm{R}^{2}=0.76$ when maturity groups were combined. This could be due to the fact that, unlike the present study, the authors included pre PHV boys in the combined regression modelling (Rumpf et al., 2015). Power related factors can influence sprinting velocity to a greater extent in pre $\mathrm{PHV}$ children due to the heightened nervous system in this phase of growth (Myer et al., 2013). Therefore, suggesting that Pmax may be a better predictor of maximal velocity in pre PHV compared to mid and post PHV boys and girls.

This present study revealed that Fo was not a strong predictor of maximal velocity even a significant difference between mid and post PHV girls existed $(\mathrm{p}<0.05)$. In contrast, Rumpf et al. (2015) reported horizontal force to be a strong predictor of maximal velocity in post $\mathrm{PHV}$ boys $\left(\mathrm{R}^{2}=0.99\right)$. A possible explanation could be that due to the differences in hormonal levels (testosterone vs. oestrogen) between genders, the force generating capacity in girls could be limited compared to their male counterparts (Malina et al., 2004). In a recent study, Coyler et al. (2020) also reported that the maximal velocity was not influenced by the force generating capacity in pre PHV girls compared to their male counterparts. In addition, the participants in this study were not trained sprinters and might have lacked the ability to apply force at the start (due to increased lean angle of the body) unlike trained young male soccer players sprinting on a non-motorised treadmill in Rumpf et al. (2015) study. Therefore, Fo was not found to be a strong predictor of maximal velocity in this study.

\section{CONCLUSION AND FUTURE DIRECTION}

This study showed that maximum sprinting velocity for 15 and $30 \mathrm{~m}$ increased across maturity groups. Of all the kinematic variables, step length was found to be significantly greater in post PHV girls compared to mid PHV girls $(p<0.05)$. With regards to kinetic variables, Pmax and Fo were significantly greater in post PHV girls compared to mid PHV girls $(p<0.05)$. Based on the regression analysis, contact time, maximal power, step frequency, leg length, and step length were found to predict maximal sprinting speed in mid and post PHV girls. However, out of all variables, contact time and power max predicted maximal velocity for both 15 and $30 \mathrm{~m}$, with contact time being the best predictor across all variables for both 15 and $30 \mathrm{~m}$. Therefore, developing these kinematic and kinetic factors associated with sprinting particularly reducing ground contact time can be beneficial in overall development of sprinting in mid and post PHV girls. Significant growth-related changes occur between the pre and mid PHV phases in youth. Future research investigating the kinetics and kinematics of sprinting speed in young females should include a pre PHV group.

\section{DISCLOSURE STATEMENT}

This is to acknowledge that there has been no financial interest or benefit that has arisen from the direct applications of this research.

\section{REFERENCES}

1. Armstrong, N., Welsman, J.R., Williams, C.A., \& Krby, B.J. (2000). Longitudinal changes in young people's short-term power output. Medicine and Science in Sports and Exercise, 32(6), 1140-5. doi: 10.1097/00005768-200006000-00017

2. Arsac, L. M., \& Locatelli, E. (2002). Modelling the energetics of $100-m$ running by using speed curves of world champions. Journal of Applied Physiology, 92(5), 1781-8.

3. Bezodis, N. E., Salo. A.I., \& Trewartha, G. (2012). Measurement error in estimates of sprint velocity from a laser displacement measurement device. International Journal of Sports Medicine, 33(6), 439-44. doi: 10.1055/s$0031-1301313$

4. Brughelli, M., Cronin, J., \& Chaouachi, A. (2011). Effects of running velocity on running kinetics and kinematics. Journal of Strength and Conditioning Research, 25(4), 933-9.

5. Buchheit, M., Samozino, P., Glynn, J. A., Michael, B. S., Haddad, H. A., Mendez-Villanueva, Morin, J-B. (2014). Mechanical determinants of acceleration and maximal sprinting speed in highly trained young soccer players. Journal of Sports Sciences, 32(20), 1-8. doi: 10.1080/02640414.2014.965191

6. Colyer, S. L., Nagahara, R., Takai, Y., \& Salo, A. I. T. (2020). The effect of biological maturity status on ground reaction force production during sprinting. Scandinavian Journal of Medicine and Science in Sports, 1-11.

7. Cross, M. R., Brughelli, M., Brown, S. R., Samozino, P., Gill, N. D., Cronin, J.B., \& Morin, J.B. (2015). Mechanical Properties of Sprinting in Elite Rugby Union and Rugby League. International Journal of Sports Physiology and Performance, 10(6), 695-702. doi: 10.1123/ijspp.2014-0151

8. Forbes, H., Bullers, A., Lovell, A., McNaughton, L.R., Polman, R.C., \& Siegler, J.C. (2009). Relative torque profiles elite male youth football players. Effects of age and pubertal development. International Journal of Sports Medicine, 30, 592-597

9. Haddad, H., Simpson, B. M., \& Buchheit, M. (2015). Monitoring changes in jump and sprint performance: best or average values? International Journal of Sports Physiology and Performance, 10(7), 931-4. doi: 10.1123/ ijspp.2014-0540

10. Lloyd, R.S., Radnor, J.M., De Ste Croix, M.B., Cronin, J.B., Oliver, J.L. (2016a). Changes in Sprint and Jump Performances After Traditional, Plyometric, and Combined Resistance Training in Male Youth Pre- and Post-Peak 
Height Velocity. Journal of Strength and Conditioning Research, 30(5), 1239-47.

11. Malina, R.M., Bouchard, C., \& Bar-Or, O. (2004). Growth, maturation and physical activity (2nd Ed). Champaign, IL: Human Kinetics.

12. Meyers, R.W., Oliver, J., Hughes, M., Cronin, J. (2015). Maximal Sprint Speed in Boys of Increasing Maturity. Pediatric Exercise Science, 27(1), 85-94. doi: : 10.1123/ pes.2013-0096

13. Meyers, R.W., Oliver, J.L., Hughes, M.G., Lloyd, R.S., \& Cronin, J.B. (2016). The Influence of maturation on sprint performance in boys over a 21-month period. Medicine of Science in Sport and Exercise, 48(12), 2555-2562.

14. Meyers, R. W., Oliver, J. L., Hughes, M. G., Lloyd, R. S., \& Cronin, J. B. (2017). Influence of age, maturity and body size on the spatiotemporal determinants of maximal sprint speed in boys. Journal of Strength and Conditioning Research, 31(4), 1009-1016.

15. Mirwald, R.L., Baxter-Jones, A.D., Bailey, D.A., \& Beunen, G.P. (2002). An assessment of maturity from anthropometric measurements. Medicine and Science in Sport and Exercise, 34(4), 689-94.

16. Morin, J.B., Jeannin, T., Chevallier, B., \& Belli, A. (2006). Spring-mass model characteristics during sprint running: correlation with performance and fatigue-induced changes. International Journal of Sports Medicine, 27(2), 158-65.

17. Morin, J. B., \& Seve, P. (2011). Sprint running performance: Comparison between treadmill and field conditions. European Journal of Physiology, 111(8), 1695-703. doi: 10.1007/s00421-010-1804-0

18. Myer, G.D., Kushner, A.M., Faigenbaum, A.D., Kiefer, A., Kashikar-Zuck, \& Clark, J.F. (2013). Training the developing brain, part 1: cognitive developmental considerations for training youth. Current Sports Medicine Reports, 12(5), 304-10.

19. Nagahara, R., Haramura, M., Takai, Y., Oliver, J., Wichitaksorn, N., Sommerfield, L.M., Cronin, J.B. (2019). Age-related differences in kinematics and kinetics of sprinting in young female. Scandinavian Journal of Medicine and Science in Sport, 29, 800-807.

20. Nilsson, J. \& Thorstensson, A. (1989). Ground reaction forces at different speeds of human walking and running. Acta Physiologica, 136(2), 217-27.

21. Nummela, A., Keranen, T., \& Mikkelsson, L.O. (2007). Factors related to top running speed and economy. International Journal of Sports Medicine, 28(8), 655-61.

22. Oliver, J., Lloyd, R., \& Rumpf, M.C. (2013). Developing speed throughout childhood and adolescence: The role of growth, maturation and training. Strength and Conditioning Journal, 35(3), 42-48.

23. Oliver, J.L., \& Stembridge, M. (2011). Use of a heart rate-toground contact time index to monitor and predict middledistance running. European Journal of Sports Science, 11(6), 431-436.

24. Phillippaerts, R.M., Vaeyens, R., Jansesens, M., Van Renterghem, B., Marthys, D., Craen, R.,....Malina, R.M. (2006). The relationship between peak height velocity and physical performance in youth soccer players. Journal of Sports Science, 24(3), 221-30.

25. Ramos, E., Frontera, W., Llopart, A., \& Feliciano, D. (1998). Strength and hormonal levels in adolescents: gender related differences. International Journal of Sports Medicine, 19, 526-31.

26. Round, J.M., Jones, D.A., Honour, J.W., \& Nevill, A.M. (1999). Hormonal factors in the development of differences in strength between boys and girls during adolescence: a longitudinal study. Annals of Human Biology, 26(1), 46-62.

27. Rumpf, M.C., Cronin, J.B., Oliver, J., \& Hughes, M. (2015). Kinematics and kinetics of maximum running speed in youth across maturity. Pediatric Exercise Science, 27(2), 277-284

28. Schepens, B., Williams, P., \& Cavagna, G. (1998). The mechanics of running in children. Journal of Physiology, 509, 927-940.

29. Simperingham, K., Cronin, J., Pearson, S., \& Ross, A. (2017). Reliability of horizontal force-velocity-power profiling during short sprint-running accelerations using radar technology. Sports Biomechanics, 18(1), 88-99. doi: 10.1080/14763141.2017

30. Talukdar, K. Harrison, C., McGuigan, M. (2021). Intraday and inter-day reliability of sprinting kinetics in young female athletes measured using a radar gun. Measurement in Physical Education and Exercise Science, 25(1), 1-7. doi: 10.1080/1091367X.2021.1876068

31. Vanderka, M. \& Kampiller, T. (2012). Age related changes of running stride kinematics in 7 to 18 year of youth. Exercise and Quality of Life, 4(1), 11-24.

32. Viru, A., Loko, J., Volver, A., Laaneots, \& Viru, M. (1999). Critical Periods in the Development of Performance Capacity During Childhood and Adolescence. European Journal of Physical Education, 4(1), 75-119. 